\title{
The Clinical Application of Skin Autofluorescence: An Indicator of Glycemic Control and Diabetic Nephropathy Progression
}

\author{
Ziwei $\mathrm{W}$ and Junqing $\mathrm{Z}^{*}$ \\ Department of endocrinology, Peking University First Hospital, China
}

*Corresponding author: Junqing Zhang, Department of endocrinology, Peking University First Hospital, 8 Xishiku Ave., 10034, Beijing, China, Tel: +86 010 83572574; Fax: +86 010 83572312; E-mail: junqing.zhang@pkufh.com

\section{Abstract}

Diabetic nephropathy has become the leading cause of end-stage renal disease worldwide, which exacerbates under poorly controlled hyperglycemia. However, the current methods in monitoring long-term glycemic control and predicting the progression of renal complications are still limited due to the relatively quick turn-over rate. Skin autofluorescence has been increasingly recognized as a convenient and stable marker in reflecting the development of diabetic complications. Compared with current parameters, skin autofluorescence can better represent the previous glycemic control in a long period of time. In addition, the strong correlation between skin autofluorescence and future impairment of renal function draws special attention. This review focuses on the recent clinical trials concerning the application of skin autofluorescence in reflecting overall AGEs accumulation and predicting the progression of diabetic nephropathy.

Keywords: Diabetic nephropathy; Skin autofluorescence; Advanced glycation end-products; Glycated haemoglobin

Abbreviations: DN: Diabetic Nephropathy; AGEs: Advanced Glycation End-products; GFR: Glomerular Filtration Rate; ELISA: Enzyme-Linked Immunosorbent Assay; AF: Autofluorescence; LC: Liquid Chromatography; MS: Mass Spectroscopy; AER: Albumin Excretion Rate; IDNS: International Diabetic Nephropathy Study; AUC: Area Under the Curve; CML: Carboxymethyl.

\section{Introduction}

Diabetic nephropathy (DN), as one of the most significant vascular complications of diabetes mellitus, has become the leading cause of end-stage renal disease worldwide. Multiple pathways have been related to underlie the mechanisms of DN, amongst which the advanced glycation end-products (AGEs) and its downstream pathway play a prominent role [1].
AGEs is a heterogeneous group of final products formed by the reaction between the reactive carbonyl groups of reducing sugars and the free amino groups of protein, lipids and nucleic acids. The non-enzymatic formation AGEs has long been believed to be a slow process, during which the initial reaction product, also called Schiff base, undergoes the structural rearrangement and finally converts into the irreversible end-products [2]. However, in recent years, remarkable progress has been made in linking the rapid formation of AGEs to carbonyl stress and oxidative stress [3-5], which are present under diabetic conditions [6].

In the human body, AGEs extensively forms cross links with long-lived proteins (e.g., with collagen) and directly deteriorates the structure of normal tissue. On the other hand, AGEs can induce the expression of its receptor 
(receptor of AGEs, RAGE), which subsequently sparks a vicious circle of oxidative stress and inflammation [7].

In the kidney, the accumulation of AGEs increases the thickness of glomerular basement membrane and induces the fibrosis of tubular area, thus directly deteriorates the kidney structure [2]. The clearance of AGEs depends majorly upon the turnover of AGEs-crossed proteins, and subsequently the degraded AGEs are excreted through the kidney. However, in the circumstances of DN, the accumulation of AGEs caused by the persisting hyperglycemia exacerbates due to the slow clearance and impaired renal function. In addition, the dramatically elevated oxidative stress [8] and carbonyl stress [9] in DN can in turn promote the formation of AGEs. The selfperpetual mechanism of AGEs adds huge complexity to the intervention of DN.

\section{Comparison of Methods in Detecting AGEs Levels}

Current methods of AGEs detection include liquid chromatography-mass spectroscopy (LC-MS) in measuring different AGEs component in the plasma [6] and skin tissue homogenates $[10,11]$, enzyme-linked immunosorbent assay (ELISA), fluorescent measurement of the autofluorescence (AF) in the plasma [12] and skin [13]. The self-emission of fluorescence is another feature of AGEs, thus allowing for non-invasive measurement of AGEs accumulation in the tissue. As mentioned, AGEs tends to crosslink with long-live proteins like collagen, which is rich in skin, therefore the skin AF can serve as a stable indicator of AGEs [14]. On the other hand, the role of plasma AGEs in reflecting overall accumulation is limited due to the relatively quick turn-over rate of serum proteins [15].

It has been reported that skin $\mathrm{AF}$, other than plasma AGEs levels, is related to the vascular function in dialysis patients [14]. Despite the accuracy of plasma AGEs levels measured by LC-MS, the adequate ability of skin AF in reflecting tissue AGEs content as a whole has been increasingly accepted. Studies also find that skin AF correlates with the AGE levels measured in skin homogenates from biopsies [14], providing the possibility of using skin $\mathrm{AF}$ as a quick clinical detecting tool.

\section{Skin AF as a Long-term Marker of DN Progression}

The application of skin AF into clinical use was established by Meearwaldt $\mathrm{R}$, et al. [16]. Using the
Autofluorescence Reader, this study found out that in diabetic patients, skin AF correlated with diabetic duration, creatinine levels and mean glycated hemoglobin (HbA1c) in previous years, validating its role in reflecting long-term glycemic control and DN development.

In 2008, Gerrits E, et al. [13] applied skin AF as a risk predictor of diabetic microvascular complications for the first time. After a median follow-up of 3.1 years, the study that contains 881 surviving patients found out that the baseline skin AF was markedly higher in patients who developed albuminuria (defined as albumin/creatinine $>2.5 \mathrm{mg} / \mathrm{mmol}$ for men and $>3.5 \mathrm{mg} / \mathrm{mmol}$ for women), an early manifestation of DN.

The Diabetes Control and Complications Trial (DCCT) compared long-term intensive treatment with conventional methods of intervening hyperglycemia in type 1 diabetic patients. The DCCT group also found in 2015 that the levels of skin AGEs are independently associated with long-term microvascular complication progression, including DN [10]. Although the skin AGEs were measured from biopsies, a strong association between skin collagen AGEs and future DN development (defined as microalbuminuria, with albumin excretion rate (AER) $\geq 40 \mathrm{mg} / 24 \mathrm{~h}$ ) up to 17 years was proved.

In terms of the association of skin $\mathrm{AF}$ and future renal function, Velayoudom-Cephise F, et al. [17] reported that after a 4-year follow-up, skin AF not only predicted macrovascular events but also the estimated glomerular filtration rate (eGFR) impairment in 243 type 1 diabetic patients. After adjustment for diabetes duration, HbA1c, AER and other diabetes-related risk factors, the inverse relation between skin AF and eGFR remained significant. The study emphasized the role of skin $\mathrm{AF}$ as a predictor in the progression of DN. In view of type 2 diabetic patients, a recent study also confirmed that skin AF also significantly increased with the severity of DN [18].

\section{Comparison between Skin AF and Other Markers in Glycemic Control}

The parameters that are currently used to assess glycemic control include the blood glucose level, HbA1c and glycated serum albumin. Blood glucose only reflects the immediate status of glucose level, which fluctuates largely in a short time. HbA1c is vastly recognized as an indicator reflecting the status of blood glucose, but it was limited by the turn-over rate of red blood cell (2-3 months). Likewise, the glycated serum albumin, the halflife period only lasts 19 days, hence only the glycemic control of 2-3 weeks can be reflected. In addition, the 
International Diabetic Nephropathy Study (IDNS) pointed out that there was only a weak association between $\mathrm{HbA1c}$ and the development of pathological renal changes in a 5-year follow-up of 243 type 1 diabetic patients. An indicator that is able to reflect long-term glycemic control is still scarce.

However, in 2013, Sugisawa E, et al. [19] compared the HbA1c area under the curve (AUC) with skin AF in analyzing the glycemic control for the past 20 years for the first time, and found that skin AF significantly correlated with $\log A C R$ and the impairment of eGFR. Despite that skin AF also correlates with HbA1c AUC, after adjustment, only skin AF remained to associate with the progression of DN. In comparison with the numerous tests of HbA1c levels that need to be measured every 3 months, skin AF possesses the advance of convenience and accuracy in monitoring long-term status of blood glucose control, and also serves as an indicator of renal impairment.

\section{The limitation of Skin AF}

The AGEs is a group of various cumulative endproducts, which accumulates not only with blood glucose level, but also with age and the intrinsic color of skin. This correlation with age and different races limits the use of skin $\mathrm{AF}$ as a diagnostic method [16]. Also, some components of AGEs that cannot be detected by fluorescence reader, such as $\mathrm{Ne}$-(carboxymethyl)-lysine (CML), take up a considerable part of AGEs. However, according to the comparative study of Meerwaldt, et al. [16], skin AF also correlated with the non-fluorescent AGEs in the skin, supporting the role of skin AF as an overall marker of AGEs accumulation. Up until now, the major subjects of clinical trial using skin $\mathrm{AF}$ as a predictor focused on the Caucasians, whereas studies upon the skin $\mathrm{AF}$ in other races are still calling for more attention $[20,21]$.

\section{Conclusion}

Skin AF, an integrative parameter that parallels with tissue accumulation of AGEs, has been increasingly recognized in reflecting glycemic control and predicting the progression of $\mathrm{DN}$, a major cardiovascular complication of diabetes mellitus. This simple, convenient and non-invasive method of estimating overall AGEs levels holds promising value in the future application of assessment of diabetic complications.

\section{References}

1. Tonna S, El-Osta A, Cooper ME, Tikellis C (2010) Metabolic memory and diabetic nephropathy: potential role for epigenetic mechanisms. Nat Rev Nephrol 6(6): 332-341.

2. Yamagishi S, Maeda S, Matsui T, Ueda S, Fukami K, et al. (2012) Role of advanced glycation end products (AGEs) and oxidative stress in vascular complications in diabetes. Biochim Biophys Acta (BBA) - General Subjects 1820(5): 663-671.

3. Matafome P, Rodrigues T, Sena C, Seica R (2017) Methylglyoxal in Metabolic Disorders: Facts, Myths, and Promises. Med Res Rev 37(2): 368-403.

4. Nigro C, Leone A, Raciti GA, Longo M, Mirra P, et al. (2017) Methylglyoxal-Glyoxalase 1 Balance: The Root of Vascular Damage. Int J Mol Sci 18(1): 188.

5. Rabbani N, Thornalley PJ (2014) The critical role of methylglyoxal and glyoxalase 1 in diabetic nephropathy. Diabetes 63(1): 50-52.

6. Beisswenger PJ, Howell SK, Russell GB, Miller ME, Rich SS, et al. (2013) Early Progression of Diabetic nephropathy correlates with Methylglyoxal-Derived Advanced Glycation End Products. Diabetes Care 36(10): 3234-3239.

7. Bierhaus A, Schiekofer S, Schwaninger M, Andrassy M, Humpert PM, et al. (2001) Diabetes-associated sustained activation of the transcription factor nuclear factor-kappaB. DIABETES 50(12): 2792-2808.

8. Thallas-Bonke V, Thorpe SR, Coughlan MT, Fukami K, Yap FYT, et al. (2008) Inhibition of NADPH oxidase prevents advanced glycation end product-mediated damage in diabetic nephropathy through a protein kinase C-alpha-dependent pathway. Diabetes 57(2): 460-469.

9. Beisswenger PJ, Drummond KS, Nelson RG, Howell SK, Szwergold BS, et al. (2005) Susceptibility to diabetic nephropathy is related to dicarbonyl and oxidative stress. Diabetes 54(11): 3274-3281.

10. Genuth S, Sun W, Cleary P, Gao X, Sell DR, et al. (2015) Skin advanced glycation end products glucosepane and methylglyoxal hydroimidazolone are independently associated with long-term microvascular complication progression of type 1 diabetes. Diabetes 64(1): 266-278. 
11. Genuth S, Sun W, Cleary P, Sell DR, Dahms W, et al. (2005) Glycation and carboxymethyllysine levels in skin collagen predict the risk of future 10-year progression of diabetic retinopathy and nephropathy in the diabetes control and complications trial and epidemiology of diabetes interventions and complications participants with type 1 diabetes. Diabetes 54(11): 3103-3111.

12. Li X, Hu J, Zhang R, Sun X, Zhang Q, et al. (2008) Urocortin ameliorates diabetic nephropathy in obese db/db mice. Br J Pharmacol 154(5): 1025-1034.

13. Gerrits EG, Lutgers HL, Kleefstra N, Graaff R, Groenier $\mathrm{KH}$, et al. (2008) Skin autofluorescence: a tool to identify type 2 diabetic patients at risk for developing microvascular complications. Diabetes Care 31(3): 517-521.

14. Hartog JW, Hummel YM, Voors AA, Schalkwijk CG, Miyata T, et al. (2008) Skin-autofluorescence, a measure of tissue advanced glycation end-products (AGEs), is related to diastolic function in dialysis patients. J Card Fail 14(7): 596-602.

15. Fraser SD, Roderick PJ, McIntyre NJ, Harris S, McIntyre CW, et al. (2014) Skin autofluorescence and all-cause mortality in stage 3 CKD. Clin J Am Soc Nephrol 9(8): 1361-1368.

16. Meerwaldt R, Graaff R, Oomen PH, Links TP, Jager JJ, et al. (2004) Simple non-invasive assessment of advanced glycation endproduct accumulation. Diabetologia 47(7): 1324-1330.

17. Velayoudom-Cephise FL, Rajaobelina K, Helmer C, Nov S, Pupier E, et al. (2016) Skin autofluorescence predicts cardio-renal outcome in type 1 diabetes: a longitudinal study. Cardiovasc Diabetol 15(1): 127.

18. Yoshioka K (2018) Skin Autofluorescence is a Noninvasive Surrogate Marker for Diabetic Microvascular Complications and Carotid IntimaMedia Thickness in Japanese Patients with Type 2 Diabetes: A Cross-sectional Study. Diabetes Ther 9(1): 75-85.

19. Sugisawa E, Miura J, Iwamoto Y, Uchigata Y (2013) Skin Autofluorescence Reflects Integration of Past Long-Term Glycemic Control in Patients with Type 1 Diabetes. Diabetes Care 36(8): 2339-2345.

20. den Hollander NC, Mulder DJ, Graaff R, Thorpe SR, Baynes JW, et al. (2007) Advanced glycation end products and the absence of premature atherosclerosis in glycogen storage disease Ia. J Inherit Metab Dis 30(6): 916-923.

21. Meerwaldt R, Hartog JW, Graaff R, Huisman RJ, Links TP, et al. (2005) Skin autofluorescence, a measure of cumulative metabolic stress and advanced glycation end products, predicts mortality in hemodialysis patients. J Am Soc Nephrol 16(12): 3687-3693. 